\title{
Testosterone metabolite nonessential for androgen effects
}

Conversion of testosterone to its metabolite $5 a$-dihydrotestosterone (DHT) is not obligatory for mediating a number of androgenic effects in adult men, say US researchers in a study published in JAMA. They suggest that testosterone and DHT mediate androgenic effects interchangeably.

Inhibitors of steroid $5 \alpha$-reductase (also known as 3-oxo-5a-steroid 4-dehydrogenase 2) suppress the conversion of testosterone to DHT and are used to treat benign prostatic hyperplasia and androgenic alopecia. DHT is known to have a developmental role during sexual differentiation; "however, the role of DHT in adult men remains unclear," says lead investigator Shalender Bhasin (Boston University School of Medicine).

Previous clinical trials have linked alterations in steroid $5 a$-reductase activity and DHT levels with adverse effects on muscle mass and sexual function. Given that steroid $5 a$-reductase inhibitors are

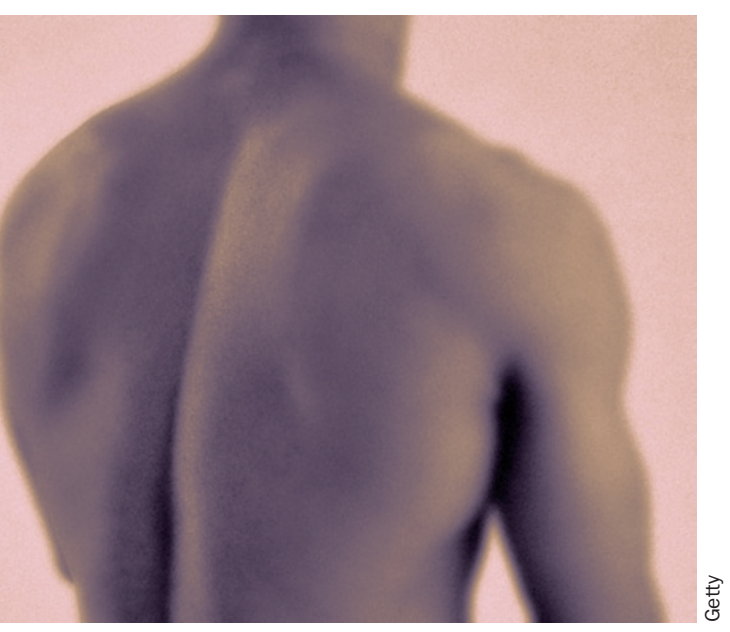

used to treat primarily older men, who are at an increased risk of reduced muscle mass and sexual dysfunction, it is important to understand the role that steroid $5 a$-reductase and DHT have in effecting these androgen-sensitive outcomes.

The researchers conducted a parallelgroup, double-blind, randomized, placebocontrolled trial, in which 139 patients with suppressed endogenous testosterone production were given graded doses of testosterone in the presence or absence of a steroid $5 a$-reductase inhibitor. Testosterone levels were directly controlled in this study; hence, treatment with steroid $5 a$-reductase inhibitors did not increase testosterone levels as in previous studies, and the role of DHT could be analyzed independently.

The researchers found that the use of steroid $5 a$-reductase inhibitors, and consequently a reduction in DHT levels, did not decrease muscle mass or increase sexual dysfunction. Although these findings indicate that these drugs are safe, results from previous studies suggest that men with low testosterone levels might experience adverse effects through the loss of the compensatory effects of DHT.

Nevertheless, "these findings bode well for the safety of steroid $5 a$-reductases," says Bhasin, "because they indicate that these drugs will not induce muscle loss".

\section{Fiona Mitchell}

Original article Bhasin, S. et al. Effect of testosterone supplementation with and without a dual $5 a$-reductase inhibitor on fat-free mass in men with suppressed testosterone production: a randomized controlled trial. JAMA 307, 931-939 (2012) 